\title{
A prospective, single-center clinical trial of a modified Cox maze procedure with bipolar radiofrequency ablation
}

Sydney L. Gaynor, MD

Michael D. Diodato

Sunil M. Prasad, MD

Yosuke Ishii, MD

Richard B. Schuessler, PhD

Marci S. Bailey, RN

Nicholas R. Damiano

Jeffrey B. Bloch, BA

Marc R. Moon, MD

Ralph J. Damiano, Jr, MD

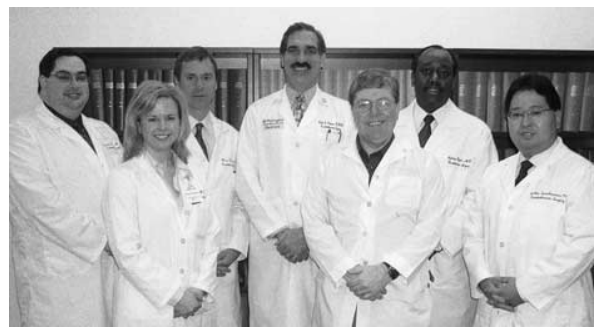

Left to right: Diodato, Bailey, Moon, R. Damiano, Schuessler, Gaynor, Ishii

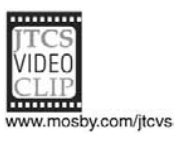

From the Division of Cardiothoracic Surgery, Washington University School of Medicine, St Louis, Mo.

Read at the Twenty-ninth Annual Meeting of The Western Thoracic Surgical Association, Carlsbad, Calif, June 18-21, 2003.

Received for publication Dec 12, 2003; revisions requested Feb 12, 2004; accepted for publication Feb 26, 2004.

Address for reprints: Ralph J. Damiano, Jr, MD, Suite 3108 Queeny Tower, 1 BarnesJewish Hospital Plaza, St Louis, MO 63110 (E-mail: damianor@msnotes.wustl.edu).

J Thorac Cardiovasc Surg 2004;128:535-42 0022-5223/\$30.00

Copyright (C) 2004 by The American Association for Thoracic Surgery

doi:10.1016/j.jtcvs.2004.02.044
Objective: The Cox maze III procedure has excellent long-term efficacy in curing atrial fibrillation. It has not been widely practiced because it is technically challenging and requires prolonged cardiopulmonary bypass. The aim of this study was to examine a simplified Cox maze III procedure that uses bipolar radiofrequency energy as an ablative source.

Methods: Beginning January 2002, a total of 40 consecutive patients underwent a modified Cox maze III procedure with bipolar radiofrequency energy. Nineteen had a lone maze procedure and 21 had a maze procedure plus a concomitant operation. One month after the operation, the first 8 patients were investigated with high-resolution magnetic resonance imaging. Patients were followed up monthly with clinical examination and electrocardiography.

Results: There was no operative deaths. The crossclamp times were $47 \pm 26$ minutes for the modified lone Cox maze III procedure and $92 \pm 37$ minutes for the Cox maze III procedure plus concomitant procedures. These were significantly shorter than our previous times for the traditional Cox maze III procedure $(93 \pm 34$ minutes and $122 \pm 37$ minutes, respectively, $P<.05$ ). Follow-up magnetic resonance imaging showed no evidence of pulmonary vein stenosis, and atrial contractility was preserved in all patients. There were no late strokes. At 6-month follow-up, $91 \%$ of patients $(21 / 23)$ were in sinus rhythm.

Conclusions: Bipolar radiofrequency ablation can be used to replace the surgical incisions of the Cox maze procedure. This energy source did not result in pulmonary vein stenosis. The modification of the Cox maze III procedure to use bipolar radiofrequency ablation simplified and shortened this procedure without sacrificing short-term efficacy.

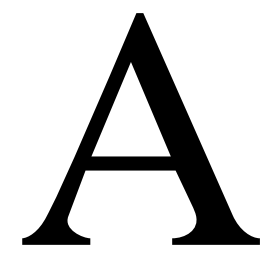

trial fibrillation (AF), the most common sustained cardiac arrhythmia, affects as much as $6 \%$ of the population older than 65 years. ${ }^{1}$ It is estimated that 1.4 million outpatient hospital visits and 227,000 hospitalizations occur annually for the treatment of $\mathrm{AF}^{2}$ Anticoagulation, rate control, antiarrhythmic drugs, cardioversion, and catheter ablation therapy all play a role in the treatment of this arrhythmia, but all of these strategies have had limited success.

The Cox maze procedure was introduced 15 years ago by James Cox for the surgical treatment of refractory AF. It was hypothesized that this operation would 


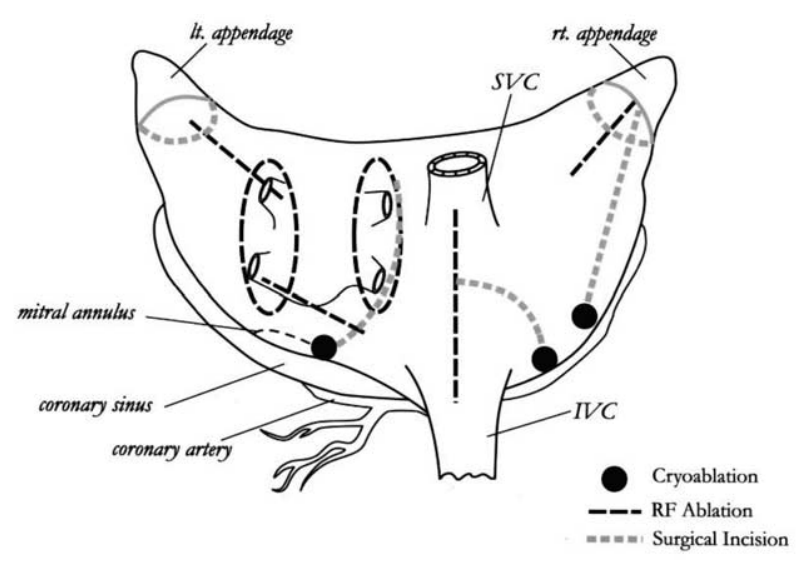

Figure 1. Modified Cox maze lesion set used in trial. IVC, Inferior vena cava; $S V C$, superior vena cava.

cure AF by abolishing the macroreentrant circuits responsible for its maintenance. This result was achieved by creating conduction block with transmural surgical incisions. Since its introduction, the operation has undergone two modifications. The Cox maze III procedure (CMP) is the most recent version and has had an impressive success rate of more than $95 \%$ at 10 years. ${ }^{3}$ The late stroke rate in our series has been less than $1 \%$, with only 1 reported event among more than 200 consecutive patients. Normally, such success would have resulted in rapid adoption of a surgical procedure. However, implementation has been slowed by its perceived invasiveness and difficulty. The traditional "cut and sew" technique requires cardiopulmonary bypass and can be time-consuming. This has resulted in a hesitation by many surgeons to combine this procedure with valve repair or replacement. Furthermore, significant morbidity has been reported, including pacemaker requirement and left atrial dysfunction. ${ }^{4,5}$

Simplification of the CMP by eliminating the traditional cut and sew approach has been proposed as a way to increase the adoption of this curative operation. Surgical investigators have used a variety of different energy sources to create lines of ablation to replace several, and in some instances all, of the myriad incisions of the CMP. Radiofrequency (RF) energy has frequently been used in the ablation of cardiac tissue..$^{6-8}$ It is capable of producing myocardial necrosis when applied to either the endocardium or epicardium. Unipolar RF energy, however, has been unable to consistently produce transmural lesions. Because the energy is unfocused, it also has the potential for unwanted collateral damage. ${ }^{9-12}$ To overcome these shortcomings, we have investigated a unique bipolar RF device. The RF energy is delivered between two closely approximated 1-mm electrodes embedded in the jaws of the clamp. By measuring the drop in conductance, transmurality of ablation can be measured on-line and used to control the time of energy delivery. Moreover, the lesions are thin and discrete, eliminating the possibility of collateral tissue injury. ${ }^{13-15}$ After extensive experimental evaluation, a prospective clinical trial was undertaken to examine the effectiveness of the bipolar RF device to replace some of the cut and sew lesions in the CMP. The objectives of this modification of the CMP were to simplify the procedure and reduce operating time while retaining the efficacy and safety of the procedure.

\section{Patients and Methods}

From January 2002 to October 2003, a total of 40 consecutive patients underwent a modified CMP with a bipolar RF device (AtriCure, Inc, Cincinnati, Ohio). More than half of the incisions of the CMP were replaced with RF ablation lines, and the device was used to individually isolate the right and the left pulmonary veins (Figure 1). This simplified the procedure by reducing the number of atrial incisions to two on the right atrium and one on the left atrium, plus amputation of the left atrial appendage. All of the procedures were performed by one surgeon (R.J.D.). Approval for this study was granted by the institutional review board at the Washington University School of Medicine in St Louis. Informed consent and approval for release of information were received from each patient.

The bipolar RF device used in this study consisted of a hand piece and a RF generator (Figure 2). The generator was capable of controlling both the voltage and the current delivered to the electrodes in the hand piece. A laptop computer with LabView version 5.1 (National Instruments Corporation, Austin, Tex) was used to monitor and record temperature, time, current, voltage, impedance, conductance, and energy continuously during ablation. The temperature of the myocardium was recorded $1 \mathrm{~mm}$ from the electrode edge. These variables were displayed in a continuous graphic format in real time.

The target tissue to be ablated was clamped between the jaws of the device. The bipolar electrodes embedded in the jaws were 5 $\mathrm{cm}$ long and $1 \mathrm{~mm}$ wide. The RF energy was delivered at $75 \mathrm{~V}$ and $750 \mathrm{~mA}$. Ablation was begun and continued until tissue conductance between the two electrodes decreased and achieved a stable minimum value for 2 seconds. This drop in conductance was used as an indicator of complete transmural ablation. Electrical isolation of the pulmonary veins was confirmed in all instances by pacing, before and after ablation, with a specially designed handheld bipolar pacing probe.

\section{Technique of the Modified Maze Procedure}

After induction of anesthesia, the patients were placed on cardiopulmonary bypass with bicaval cannulation. Initially, temperature was maintained at $36^{\circ} \mathrm{C}$ to encourage sinus rhythm and allow accurate determination of pacing thresholds. The left and right pulmonary veins were dissected and surrounded with umbilical tape. Patients in AF were electrically cardioverted. Pacing thresholds were recorded from the right and the left pulmonary veins. The bipolar RF device was then placed around first the right and then the left pulmonary vein (Figure 3). The device was clamped twice to ensure complete encirclement of both the superior and the inferior veins. Occasionally, in patients with large pulmonary veins, it was necessary to clamp each vein separately. Electrical 


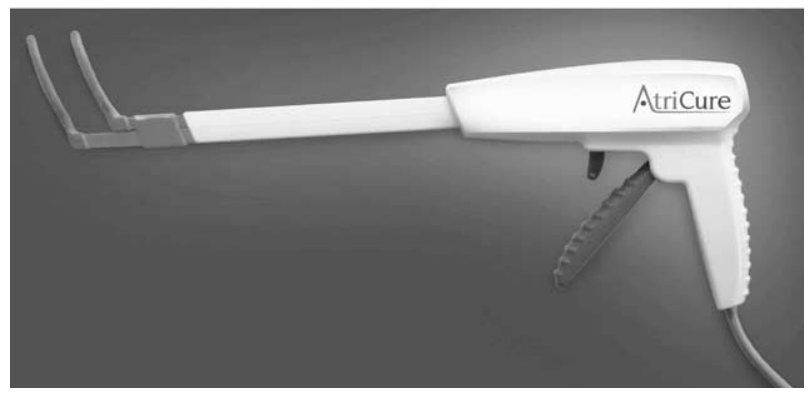

Figure 2. Bipolar RF device (AtriCure, Inc, Cincinnati, Ohio).

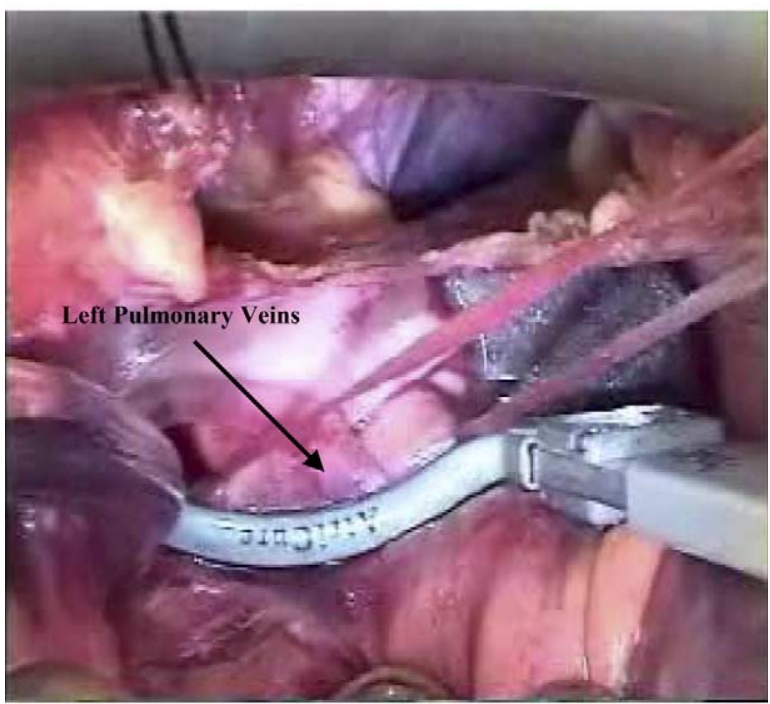

Figure 3. Bipolar RF clamp around left pulmonary veins.

isolation was documented by pacing at a stimulus strength of 20 $\mathrm{mA}$. Further ablations were performed if necessary until there was documented conduction block.

The right atrial lesions of the modified CMP were then performed. As opposed to the traditional CMP, our current modifications preserve the right atrial appendage. Two of the right atrial lesions were replaced with bipolar RF ablation lines. The right atrial free wall lesion from the right atrial appendage was made by bipolar ablation, as was the lesion between the inferior and the superior venae cavae (Figures 1 and 4; online-only video). Except for the isolation of the pulmonary veins, all ablations were done with a single application.

After completion of the right atrial lesions, a retrograde cardioplegia catheter was placed under direct vision into the coronary sinus. The heart was arrested with a combination of antegrade and retrograde cold blood cardioplegia. A standard left atriotomy was performed below the interatrial groove and extended inferiorly around the right inferior pulmonary vein (Figure 5). It was critical that this incision intersected the encircling right pulmonary vein ablation. The transseptal incision of the CMP was replaced with a bipolar RF ablation across the atrial septum onto the fossa ovalis. The atriotomy was extended inferiorly across the posterior left

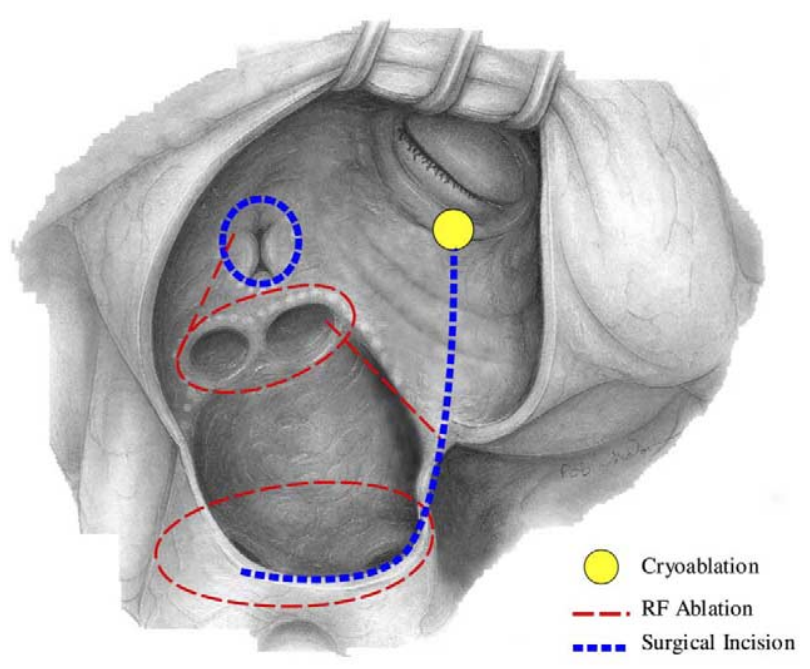

Figure 4. Left atrium with left-sided maze lesions.

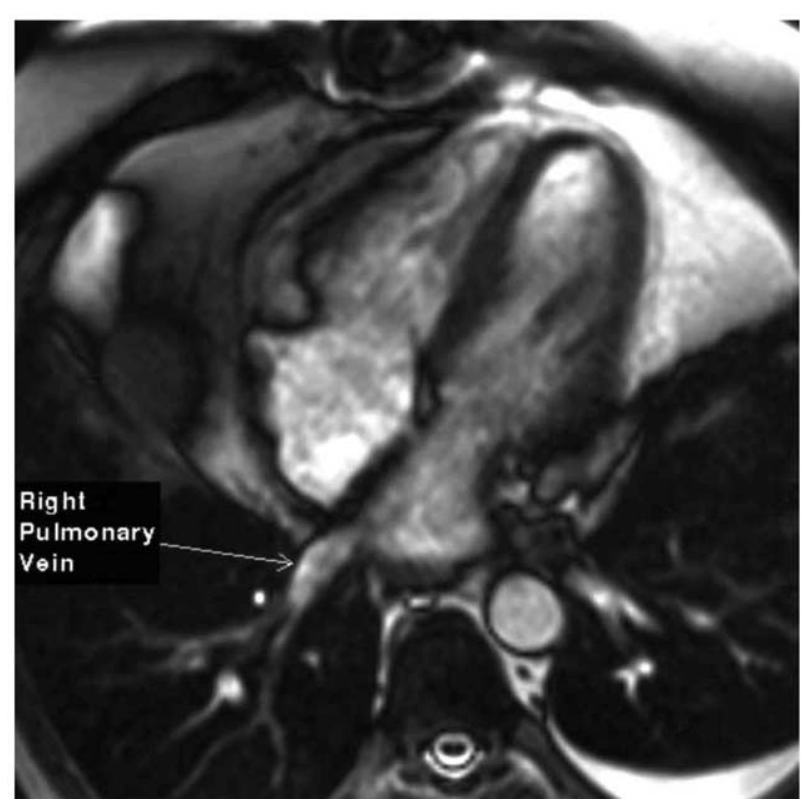

Figure 5. Four-chamber view of heart with MRI 1 month after modified CMP.

atrial free wall in the direction of the mitral valve annulus to approximately the midpoint of the P2 scallop of the posterior leaflet of the mitral valve. This incision was carried down to the atrioventricular groove and then extended endocardially with a scalpel toward the mitral annulus. This endocardial incision crossed the coronary sinus, and care was taken to avoid injury. The coronary sinus was cryoablated at $-60^{\circ} \mathrm{C}$ with a $15-\mathrm{mm}$ cryoprobe (Frigitronics Cardiac Cryosurgical System, Trumbull, Conn). The retrograde cardioplegia cannula was pulled back during this portion of the procedure. A separate 3-mm cryolesion was placed adjacent to the mitral valve annulus. A bipolar RF ablation was 
then performed from the posterior aspect of this incision, extending into the left inferior pulmonary vein (Figure 5). The left atriotomy was closed with a running monofilament suture. The heart was retracted, and the left atrial appendage was amputated. A final bipolar RF ablation was performed between the left atrial appendage and the left superior pulmonary vein. The left atrial appendage was then oversewn. The aorta was unclamped, and the right atrial lesions were closed during rewarming.

After the operation, all patients were monitored continuously for arrhythmias. Perioperative AF was treated with either intravenous or oral amiodarone. If the drug was not tolerated, patients were managed with rate control medication and elective cardioversion at 2 to 6 weeks.

One month after discharge from the hospital, the first 8 patients underwent magnetic resonance imaging (MRI) to assess for pulmonary vein stenosis and atrial function. Follow-up electrocardiograms were obtained at 1,3 , and 6 months for all patients. All patients reporting palpitations or with documented atrial arrhythmias by electrocardiography underwent 24 -hour Holter monitoring.

\section{MRI Imaging Protocol}

Subjects were placed supine in a clinical 1.5-T MRI scanner (NT Inter CV; Philips Medical Systems, Best, The Netherlands) The imaging protocol comprised multiple anatomic and functional cine images and multiple velocity-encoded images. The cine images, prospectively triggered to each $\mathrm{R}$-wave peak, were based on a steady-state gradient echocardiographic technique, balance fast field echo. Vertical long-axis cine images were obtained by aligning the left ventricular apex with the center of the mitral valve. From this vertical long-axis image, the horizontal long-axis plane was aligned through the midpoint of the left ventricular apex and the mitral valve. A diastolic image at end-expiration provided the reference image from which a stack of contiguous short-axis slices was positioned. Slice thickness was $9 \mathrm{~mm}$, and it took 8 to 10 slices to completely traverse the entire left atrium. The typical field of view was $288 \times 360 \mathrm{~mm}$ with matrix size $154 \times 256$, giving a typical reconstructed pixel size of approximately $1.5 \times 1.2 \mathrm{~mm}$.

\section{Quantitative Flow Study}

Transmitral and transtricuspid blood flows were assessed with velocity-encoded MRI. ${ }^{16}$ Imaging was centered on the mitral valve plane, with the velocity-encoded imaging slice being $7 \mathrm{~mm}$ thick. Images were acquired at 15 to 20 phases during the cardiac cycle, depending on the heart rate. Retrospective gating was used to acquire data for $20 \%$ longer than the expected R-R interval.

\section{Data Analysis}

Analysis of the acquired images was performed off-line with a commercial software package (EasyVision release 5, Philips Medical System). The volume of the left atrium was assessed by planimetry.

Analysis of quantitative flow study was performed by examining the velocity-encoded images at all time points. The peak velocity data from within these regions of interest were used to construct a velocity versus time curve, from which $\mathrm{E}$ and $\mathrm{A}$ waves could be identified. The data was then exported to a spreadsheet format for further data analysis. MRI images were used to assess anatomic and physiologic flow patterns in the pulmonary venous system. Basic cine images were used to define pulmonary venous anatomy in the short axis view. Velocity-encoded MRI images were used to quantify both direction and speed of pulmonary venous flow.

\section{Results}

\section{Operative Results}

The indications for surgery in this group included arrhythmia intolerance $(\mathrm{n}=8)$, antiarrhythmic drug failure $(\mathrm{n}=$ $20)$, recurrent thromboembolism $(\mathrm{n}=2)$, and concomitant cardiac procedures $(n=10)$. The male/female ratio was $23: 17$. The mean age was $62 \pm 12$ years. The mean duration of AF was $6.6 \pm 6.8$ years. The median duration was 3.8 years (0.5-28 years). Twenty-five patients had paroxysmal $\mathrm{AF}$, and 15 patients had persistent AF. In 35 of 40 patients $(87 \%)$ the AF was permanent. Nineteen patients underwent a lone maze procedure and 21 patients underwent the maze procedure with another concomitant cardiac operation. In this later group, 9 patients underwent the maze procedure plus mitral valve repair or replacement, 5 patients underwent the maze procedure plus coronary artery bypass grafting, and 3 patients underwent the maze procedure with coronary artery bypass grafting and a mitral valve repair.

\section{Pulmonary Vein Isolation}

Pacing thresholds for the right and left pulmonary veins were $0.9 \pm 1.0 \mathrm{~mA}$ and $1.1 \pm 1.6 \mathrm{~mA}$, respectively. The left and right pulmonary veins were isolated in every instance, as documented by pacing at $20 \mathrm{~mA}$. The right pulmonary veins underwent an average of $3.0 \pm 1.2 \mathrm{RF}$ applications, whereas the left pulmonary veins underwent $3.1 \pm 1.3 \mathrm{RF}$ applications. The mean total ablation times were $28 \pm 13$ seconds for the right pulmonary veins and $28 \pm 12$ seconds for the left pulmonary veins.

\section{Perioperative Results}

The mean RF ablation time was $9.5 \pm 3.8$ seconds for each application. The mean crossclamp time was $54 \pm 27$ minutes for the lone modified CMP. In comparison, the crossclamp time for lone traditional CMP was $93 \pm 34$ minutes $(P<.001)$. For the concomitant procedure, the average crossclamp time was $99 \pm 30$ minutes. In the traditional concomitant procedure group, the crossclamp time was 123 \pm 36 minutes $(P=.005)$.

The median intensive care unit stay was 2 days. The median hospital stay was 12 days.

\section{Postoperative Complications}

There were no operative deaths. Five patients had reexploration for mediastinal bleeding. There were no strokes or sternal infections. Postoperative AF was present in 25 patients $(63 \%)$, but this was usually transient. Thirty-four patients $(85 \%)$ were discharged from the hospital in normal 


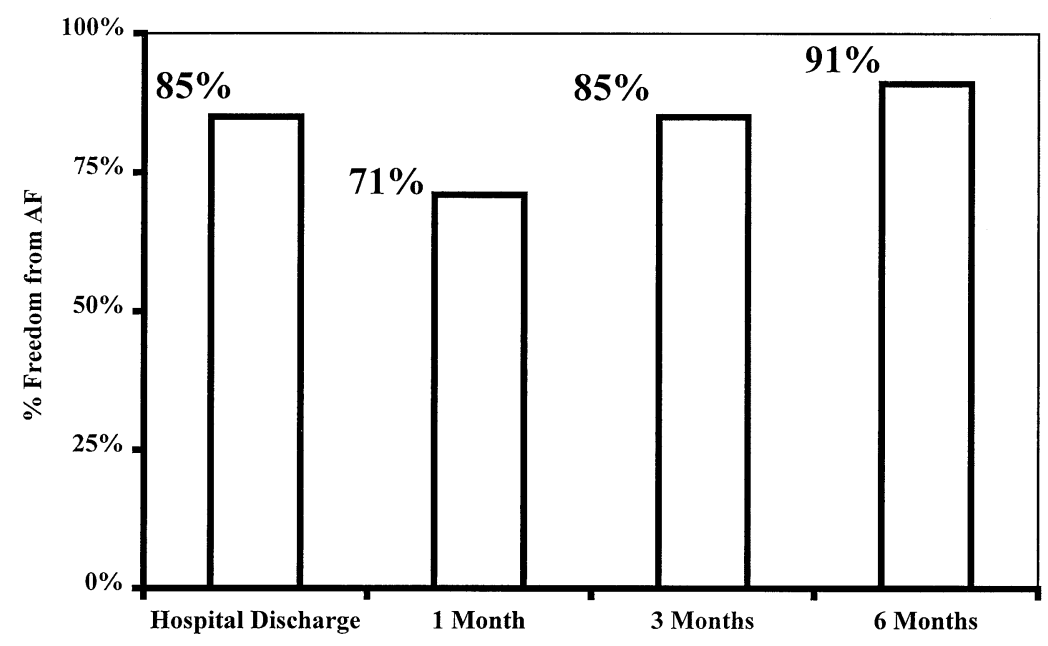

Figure 6. Freedom from AF after modified CMP.

sinus rhythm. Six patients $(15 \%)$ required postoperative permanent pacemaker placement for sick sinus syndrome. There were no complications attributable to the bipolar device in terms of collateral tissue injury.

\section{MRI Results}

MRI studies were conducted on the first 8 patients 4 to 6 weeks after operation. There was no evidence of pulmonary vein stenosis or thrombosis in any patient (Figure 6). Atrial contractility, as documented by the presence of an A wave on the transmitral flow trace, was preserved in all patients.

\section{Arrhythmia Control}

The mean follow-up time was $8.1 \pm 5.5$ months. Median follow-up was 6 months. At 1 month after the operation, 27 of 38 patients $(71 \%)$ were free from AF. At 3 months and 6 months, 28 of 33 patients $(85 \%)$ and 21 of 23 patients (91\%), respectively, were free from AF. At last follow-up, 37 of 40 patients $(93 \%)$ were free from AF. A significant decline in the number of patients requiring antiarrhythmic drug therapy was observed during a 6-month period; 32 of 40 patients $(80 \%)$ required therapy at discharge versus 10 of 23 patients $(44 \%)$ at 6-month follow-up.

\section{Discussion}

The Cox maze procedure was developed to restore normal sinus rhythm, allow for the discontinuation of anticoagulation and antiarrhythmic drugs, and reduce stroke risk. ${ }^{17,18}$ The final iteration of this operation, the CMP, was introduced in 1988. Between January 1988 and January 2002, a total of 198 consecutive patients underwent the CMP at Barnes-Jewish Hospital. ${ }^{19}$ The freedom from recurrent AF at 14 years was $93 \%$ for the entire group. The excellent long-term results with this operation established it as the criterion standard for the surgical treatment of AF.
Despite these excellent results, the CMP had shortcomings. Although mortality rates remained low $(1.5 \%$ in our series), there was significant morbidity. The operation required a relatively long period of cardiopulmonary bypass and an arrested heart. At our institution, the average crossclamp time to perform a lone maze procedure was $93 \pm 34$ minutes. After CMP, $15 \%$ of the patients required postoperative pacemakers. The median stay was 9 days after a lone CMP and 12 days after a CMP concomitant with other surgery. Finally, left atrial dysfunction has been noted in many patients. $4,20,21$

These problems with the traditional CMP have led our group and others to begin evaluating strategies to simplify the surgical treatment of AF. ${ }^{22,23}$ A common strategy has been to replace the surgical incisions with linear lines of ablation made by mans of various energy sources, including RF, cryoablation, microwave, ultrasound, and laser energy. ${ }^{24,25}$ Elimination of the extensive cutting and sewing needed to make the atrial incisions of the CMP gives these new operations the potential to simplify the procedure and decrease the time of surgery. The hope is that this will reduce morbidity, encourage referrals, and speed the adoption of this operation by surgeons.

Many groups also have chosen to omit many of the incisions of the Cox maze procedure. This has made interpretation clinical results difficult, because failure could be due either to inadequate ablation technology or to an inadequate lesion set. It was our goal to perform all the basic lesions of the CMP but to replace many of the incisions with lines of ablation.

Our group has concentrated on a device that uses bipolar RF ablation. By measuring the fall in tissue conductance, this device was shown to have a definitive and verifiable end point of transmurality. ${ }^{13-15}$ Mean ablation times were al- 
ways less than 10 seconds, even in thick tissue, and the lesions were only 1 to $3 \mathrm{~mm}$ in width. This was an improvement relative to the width of lesions and time required for ablation with unipolar devices. This report represents our first clinical series with this device and details our operative technique and early clinical results. The safety of this technology for pulmonary vein isolation was evaluated by MRI scans at 1 month.

Our modification of the CMP replaced more than half of the surgical incisions with bipolar ablation lines. This simplified procedure has a single long left atriotomy and only two right atrial incisions. Isolation of the pulmonary veins can be performed on the beating heart before crossclamping. The use of this device decreased mean crossclamp time in both lone CMP and concomitant CMP groups by more than 30 minutes. However, it should be noted that this comparison is with a historical cohort performed by different surgeons, making direct comparison problematic.

This procedure may have other advantages relative to the cut and sew procedure in addition to its technical simplicity. With separate isolation of the right and left pulmonary veins, a significant amount of functioning posterior atrial myocardium remains, theoretically improving atrial contractility. In the traditional CMP, the right and left pulmonary veins are isolated en bloc, removing virtually all function of the entire posterior left atrium. Although it is hoped that such advantages will translate into decreased morbidity, a much larger cohort of patients will be needed to prove this hypothesis. There were no operative deaths in this series, and at 6 months $91 \%$ of patients were free from AF, with only 10 patients requiring antiarrhythmic therapy.

This device was particularly effective in isolating the pulmonary veins, a key part of any procedure for AF. The device isolated the right and left pulmonary veins in every instance. Unfortunately, the device usually required several applications to isolate the atrial cuff around the pulmonary veins. We believe that this was due to the large amount of atrium clamped during this maneuver, which can result in poor clamp apposition. Experience with the device has supported not bunching the atria into the clamp to perform a single ablation, but rather coming across the atrial cuff from both above and below in cases where there appears to be excess tissue. Although several ablations were often required, total combined ablation time for both the right and left pulmonary veins was under 60 seconds. Isolation was documented in every instance by pacing. It is our belief that surgeons who use this technology should routinely use pacing to confirm isolation.

The question of late pulmonary vein stenosis remains an important issue. In the electrophysiologic laboratory, there has been a defined incidence of late pulmonary vein stenosis. ${ }^{26,27}$ Few surgical groups have rigorously evaluated patients for this problem after RF ablation. In this study, the first 8 patients were evaluated with high-resolution MRI scans at 1 month. At that interval there was no evidence of pulmonary vein stenosis, normal pulmonary vein flow patterns were maintained, and left atrial function was preserved.

Preliminary reports from a number of electrophysiologic laboratories have suggested that pulmonary vein isolation alone may be curative for selected patients with paroxysmal AF. ${ }^{28}$ Although it still remains unclear whether this simple procedure will be effective in surgical cases, particularly those of patients with valvular heart disease, this technology does allow pulmonary vein isolation to be performed quickly, without the need for any incisions or cardiopulmonary bypass.

The use of bipolar RF ablation represents a step toward a less invasive maze procedure. Results in our research laboratory have shown that this technology can be effectively used on the beating heart. ${ }^{14}$ If the excellent early results of the modified Cox maze procedure stand the test of time, the indications for this operation should be expanded. In our own practice, we believe that the operation is a viable alternative to a lifetime of anticoagulation for young patients with solitary AF. Moreover, this simplified operation should be offered to all patients undergoing valvular surgery with concomitant AF.

This study has limitations. It represents a relatively small number of patients with short follow-up. Pulmonary vein stenosis was evaluated at 1 month, which may be too short an interval to pick up this complication. Careful long-term observation will be needed to demonstrate the late efficacy of this new modification of the CMP. The strengths of this study are that it is a prospective study of a consecutive group of patients with $100 \%$ follow-up. It is the only study to date to examine postoperative pulmonary vein stenosis after bipolar RF ablation. In summary, this study suggests that bipolar RF ablation is safe and can effectively replace the surgical incisions of the CMP.

\section{References}

1. Furberg CD, Psaty BM, Manilo TA, Gardin JM, Smith VE, Rautaharju PM. Prevalence of atrial fibrillation in elderly subjects (the Cardiovascular Health Study). Am J Cardiol. 1994;74:236-41.

2. National Center for Health Statistics. Public use data tape documentation: 1995 National Ambulatory Medical Care Survey. Hyattsville (MD): National Center for Health Statistics, US Public Health Service; 1997.

3. Cox JL, Ad N, Palazzo T, Fitzpatrick S, Suyderhoud JP, DeGroot KW, et al. Current status of the maze procedure for the treatment of atrial fibrillation. Semin Thorac Cardiovasc Surg. 2000;12:15-9.

4. Lonnerholm S, Blomstrom P, Nilsson L, Blomstrom-Lundqvist C. Atrial size and transport function after the maze III procedure for paroxysmal atrial fibrillation. Ann Thorac Surg. 2002;71:107-11.

5. Schaff HV, Dearani JA, Daly RC, Orszula TA, Danielson GK. Coxmaze procedure for atrial fibrillation: Mayo Clinic experience. Semin Thorac Cardiovasc Surg. 2000;12:30-7.

6. Jazayeri MR, Hempe SL, Sra JS, Dhala AA, Blanck Z, Deshpande SS, et al. Selective transcatheter ablation of the fast and slow pathways 
using radiofrequency energy in patients with atrioventricular nodal reentrant tachycardia. Circulation. 1992;85:1318-28.

7. Jais $P$, Shah DC, Haissaguerre M, Takahashi A, Lavergne T, Hocini $\mathrm{M}$, et al. Efficacy and safety of septal and left-atrial linear ablation for atrial fibrillation. Am J Cardiol. 1999;84(9A):139R-46R.

8. Dagres N, Clague JR, Lottkamp H, Hindricks G, Breithardt G, Borggrefe $\mathrm{M}$. Impact of radiofrequency ablation of accessory pathways on the frequency of atrial fibrillation during long-term follow-up: high recurrence rate of atrial fibrillation in patients older than 50 years of age. Eur Heart J. 2001;22:423-7.

9. Thomas SP, Guy DJ, Boyd AC, Eipper VE, Ross DL, Chard RB. Comparison of epicardial and endocardial linear ablation using handheld probes. Ann Thorac Surg. 2003;75:543-8.

10. Damiano RJ. Alternative energy sources for atrial ablation: judging the new technology. Ann Thorac Surg. 2003;75:329-30.

11. Fayad G, Le Tourneau T, Modine T, Azzaoui R, Larrue B, Koussa D, et al. One-year clinical and echocardiographic follow-up after endocardial radiofrequency ablation for atrial fibrillation. $J$ Am Coll Cardiol. 2003;41(6 Suppl B):499.

12. Gillinov AM, Petterson G, Rice TW. Esophageal injury during radiofrequency ablation for atrial fibrillation. J Thorac Cardiovasc Surg. 2001;122:1239-40.

13. Prasad SM, Maniar HS, Schuessler RB, Damiano RJ Jr. Chronic transmural atrial ablation by using bipolar radiofrequency energy on the beating heart. $J$ Thorac Cardiovasc Surg. 2002;124:708-13.

14. Prasad SM, Maniar HS, Moustakidis P, Schuessler RB, Damiano RJ Jr. Epicardial ablation on the beating heart: progress towards an off-pump maze procedure. Heart Surg Forum. 2002;5:100-4.

15. Prasad SM, Maniar HS, Diodato MD, Schuessler RB, Damiano RJ. Physiological consequences of bipolar radiofrequency energy on the atria and pulmonary veins: a chronic animal study. Ann Thorac Surg. 2003;76:836-42.

16. Naylor GL, Firmin DN, Longmore DB. Blood flow imaging by cine magnetic resonance. J Comput Assist Tomogr. 1986;10:715-22.

17. Cox JL, Schuessler RB, D'Agostino HJ Jr, Stone CM, Chang BC, Cain $\mathrm{ME}$, et al. The surgical treatment of atrial fibrillation: development of a definitive surgical procedure. J Thorac Cardiovasc Surg. 1991;101: 569-83.

18. Cox JL, Sundt TM 3rd. The surgical management of atrial fibrillation. Annu Rev Med. 1997;48:511-23.

19. Prasad SM, Maniar HS, Camillo CJ, Schuessler RB, Boineau JP, Sundt TM, et al. The Cox maze III procedure for atrial fibrillation: long-term efficacy in patients undergoing lone versus concomitant procedures. J Thorac Cardiovasc Surg. 2003;126:1822-8.

20. Ishii Y, Takashi N, Masahiro F, Ogasawara H, Iwaki H, Ohkubo N, et al. Serial change in the atrial transport function after the radial incision approach. Ann Thorac Surg. 2001;71:572-6.

21. Yashima N, Nasu M, Kawazoe K, Hiramori K. Serial evaluation of atrial function by Doppler echocardiography after the maze procedure for chronic atrial fibrillation. Eur Heart J. 1997; 18:496-502.

22. Hindricks G, Mohr FW, Autschbach R, Kottkamp H. Antiarrhythmic surgery for treatment of atrial fibrillation-new concepts. Thorac Cardiovasc Surg. 1999;47(Suppl 3):365-9.

23. Knaut M, Tugtekin SM, Spitzer S, Gulielmos V. Combined atrial fibrillation and mitral valve surgery using microwave technology. Semin Thorac Cardiovasc Surg. 2002;14:226-31.

24. Knaut M, Spitzer SG, Karolyi L, Ebert H, Richter P, Tugtekin SM, et al. Intraoperative microwave ablation for curative treatment of atrial fibrillation in open heart surgery: the MICRO-STAF and MICROPASS pilot trial. MICROwave Application in Surgical treatment of Atrial Fibrillation. MICROwave Application for the Treatment of Atrial Fibrillation in Bypass-Surgery. Thorac Cardiovasc Surg. 1999; 47(Suppl 3):379-84.

25. Gillinov AM, Smedira NG, Cosgrove DM 3rd. Microwave ablation of atrial fibrillation during mitral valve operations. Ann Thorac Surg. 2002;74:1259-61.

26. Yu WC, Hsu TL, Tai CT, Tsai CF, Hsieh MH, Lin WS, et al. Acquired pulmonary vein stenosis after radiofrequency catheter ablation of paroxysmal atrial fibrillation. J Cardiovasc Electrophysiol. 2001;12: 887-92.

27. Arentz T, Jander N, von Rosenthal J, Blum T, Furmaier R, Gornandt
L. Incidence of pulmonary vein stenosis 2 years after radiofrequency catheter ablation of refractory atrial fibrillation. Eur Heart J. 2003;24: 963-9.

28. Haissaguerre M, Jais P, Shah DC, Takahashi A, Hocini M, Quiniou G, et al. Spontaneous initiation of atrial fibrillation by ectopic beats originating in the pulmonary veins. $N$ Engl J Med. 1998;339:659-66.

\section{Discussion}

Dr Roger C. Millar (Salt Lake City, Utah). Dr Damiano and I have a similar interest in the AF problem that dates back really to Dr Cox, who was a predecessor of both of us at Duke University.

Nine months ago we became interested in this same energy device, the bipolar RF, and have performed 35 cases, all concomitant procedures. Eighty-six percent had mitral valve surgery. In that group we had 3 triple valves and 16 double valves. You have averaged 3.2 and 3.3 applications on the pulmonary veins on the right or the left to prove transmurality by atrial pacing, or technically really pulmonary vein pacing. Why do you think this is, and why do you think you required three applications to obtain complete isolation of the pulmonary veins?

Dr Damiano. That's an absolutely excellent question. Part of it has been the learning curve with the technology. First of all, we basically are trying to replicate our animal work, where we would try in just one clamp to try to bunch all the pulmonary veins into what is only a 5-cm long clamp and take a big cuff of left atrium, because we were trying to make sure we didn't see any pulmonary vein stenosis. What we have learned and what I think is happening is that if you bunch what is actually quite a bit of atrial tissue into this clamp that if you get any type of folding over of the tissue and any air in there, because air is an incredibly great insulator, you will get a false high reading of tissue resistance, with the inverse of that being a low reading of the conductance. That will, I think in human subjects give you a false-positive indication. It is actually a real indication that conductance has fallen to a stable minimum, but that is probably due to having some air or bunched up tissue in the clamp. What we have gone to in the last group of patients is that if the actual width of those pulmonary veins is more than 2 thirds of the clamp width, in which the electrode length is $5 \mathrm{~cm}$, we will then clamp the right superior and inferior and the left superior and inferior veins separately, or we will take clamps that go from both below and above. So by definition, you would have to have two clamps. By doing that we virtually always have conduction block after just two applications, but certainly in the beginning we had as many as five applications before we had complete conduction block. I would say that I certainly believe because of that that it is absolutely mandatory if you are using this technology to pace or record pulmonary vein electrograms to document that you have pulmonary vein isolation, because one would certainly consider that among the most important parts of any surgical treatment of AF.

Dr Millar. That seems to imply, then, that we need to be doing this on the beating heart, at least so we can test transmurality before proceeding with the other connecting lesions in the left atrium.

When you are doing your linear lesions to connect the pulmonary veins across the base of the left atrium, or even on the right side the superior to inferior vena cava longitudinal lesion that we construct with the bipolar device, do you do more than one burn in 
those areas because you cannot prove transmurality by pacing across those lines?

Dr Damiano. Usually not. I usually just do one burn. You can directly observe the ablation, and what we have learned in a couple of years of animal experimentation with this is that if you have uniformly thick tissue and encompass it without any bunching in this device, it is virtually $100 \%$ reliable in terms of creating a transmural lesion. If for any reason I am going over any type of tissue that appears to be a bit nonhomogeneous in terms of its thickness, to do a second ablation line doesn't take long. If I have any question in my mind, a lot of times I will put two ablation lines right there and still be done in 10 or 15 seconds. Generally for the other lines, we will perform simply one; especially along the right atrial free wall and in the posterior left atrium, the tissue is almost always very uniform in thickness, and usually just one ablation line is used in those areas.

Dr Millar. Related to this same issue, when I have placed a second burn, I have usually released the clamp and then reapplied it. It still takes almost the same amount of time. If this is full thickness to start with, why does it take several seconds again? Why, if it was transmural the first time, does it take time to signal transmurality?

Dr Damiano. The only explanation I can think of is if there is any moisture that would slip in after your ablation. Basically, the low conductance is due to a complete desiccation of the tissue. If you stop, perhaps there is some leeching in of interstitial fluid which you then have to boil off again. I probably would take that up with some of the engineers at the company. That is an interesting observation. I have never really tried that myself.

Dr Millar. Try it some time. I really can't explain it either, but that's my best explanation as well. Dr Gillinov presented a series of 190 patients undergoing left-sided procedures only and showed a 6-month $80 \%$ success rate. I noticed that in your series more than half of your patients had paroxysmal AF. Would pulmonary vein isolation and left atrial appendage removal also have given you an $80 \%$ success rate? What does that imply for the possible future application of such a device to do pulmonary vein isolation and left atrial appendage removal in less than 10 minutes? That could easily be added to any kind of left-sided operation you are doing. Would you speculate on the future?

Dr Damiano. I will speculate on the role of pulmonary vein isolation, both today and in the future. Certainly, there are provocative electrophysiologic data indicating that pulmonary vein isolation may be effective treatment for paroxysmal AF. I would caution all surgeons that the data from Dr Haissaguerre's laboratory in Bordeaux, from Dr Chen's laboratory in Taiwan, from the group in Michigan, and from Dr Morady that have justified that rapid conclusions are virtually all in patients without structural heart disease and very small left atria. So the role of pulmonary vein isolation and its possible success will probably be best determined by our surgical results and not necessarily by what has been reported in the electrophysiology literature. I think it is still a hypothesis and that we need to design good clinical studies to answer this. Clearly, with this device total ablation time to isolate both sides of the pulmonary vein was less than a minute. It can dissect, and the dissection of both the left and right sides can be performed in a couple of minutes each once you are used to doing it. We do perform this on the beating heart before crossclamping, as you have suggested, so that we can pace, and that would probably only add 5 minutes to the operation. I think that you have to amputate the left atrial appendage, although what our success rate will be in that group is unclear. Dr Gillinov reported a success rate clearly much lower than our success rate with the maze procedure. Their latest reported success is about $75 \%$. They do have a drawback in that most of their patients really have not been able to be followed up, because they usually come from a long way off. Thus I don't think we know their success rate clearly, and I think we have to look at. Even in this study, I would tell you it is too soon. I am not sure what our success rate is going to be. It is promising that we are at $95 \%$ at 6 months, but I don't know what we will be long term. We have done 10 cases with just pulmonary vein isolation. This was a highly selected group. Our success rate at 6 months has only been $50 \%$, although among patients with paroxysmal AF it was $66 \%$, so I think there is some indication that there are some patients for whom it is going to work. I think we need to get a little bit smarter, and I think we are going to have to wait for our experience to determine who these patients are. I don't think I would jump to the conclusion right now that all patients with paroxysmal AF who are referred for surgical treatment of AF should only have pulmonary vein isolation. I think that is a question remaining to be answered, and I do feel strongly that for patients who are good candidates for a CMP or a modified procedure like the one I've shown today, that's what you should do. It historically has a 10-year efficacy rate greater than $90 \%$. Now that it has been modified with bipolar ablation and some other technologies, it really does not add significantly to the procedure nor does it require a lot of lines. I do think that there certainly is going to be a group of patients who can be successfully treated with pulmonary vein isolation. I just heard a report from Dr Nitta's group in Tokyo, where they have done intraoperative mapping; they found the patients with pulmonary vein foci-interestingly not all patients with paroxysmal AF, but about $60 \%$ to $70 \%$ of those patients- underwent pulmonary vein isolation with excellent results. So that may be where we are going in the future. Certainly I think that in those cases it will play a large role and probably can be done in a minimally invasive and rapid manner by surgeons. 